\title{
Synthesis and styrene copolymerization of novel fluoro and iodomethoxy ring-disubstituted isobutyl phenylcyanoacrylates
}

Magen A. Higgs, Kelly C. Ho, Kristin N. Huffman, Alexis L. Johnson, Paulina Kapica, Melanie A. Knight, Ashley Lehan, Christina Guyn, Briana N. Hryhorysak, Sara M. Rocus, William S. Schjerven and Gregory B. Kharas

DePaul University, Chemistry and Biochemistry Department, 1110 West Belden Avenue, Chicago, IL 60614-3214

Contact: gkharas@depaul.edu

\begin{abstract}
Novel ring-disubstituted isobutyl phenylcyanoacrylates, $\mathrm{RPhCH}=\mathrm{C}(\mathrm{CN}) \mathrm{CO}_{2} \mathrm{CH}_{2} \mathrm{CH}\left(\mathrm{CH}_{3}\right)_{2}$ (where $\mathrm{R}$ is 2-fluoro-3-methoxy, 2-fluoro-4-methoxy, 2-fluoro-5-methoxy, 2-fluoro-6methoxy, 3-fluoro-4-methoxy, 4-fluoro-3-methoxy, 5-fluoro-2-methoxy, 3-iodo-4-methoxy, 5-iodo-2-methoxy) were synthesized by the piperidine catalyzed Knoevenagel condensation of ring-disubstituted benzaldehydes and isobutyl cyanoacetate and characterized by CHN analysis, IR, ${ }^{1} \mathrm{H}$ and ${ }^{13} \mathrm{C} \mathrm{NMR}$. The acrylates were copolymerized with styrene in solution with radical initiation $(\mathrm{ABCN})$ at $70^{\circ} \mathrm{C}$. The compositions of the copolymers were calculated from nitrogen analysis.
\end{abstract}




\section{Introduction}

Fluoro ring-substituted methyl phenylcyanoacrylates (PCA) were cited in such applications as pentanidium-catalyzed direct assembly of vicinal all-carbon quaternary stereocenters through $\mathrm{C}(\mathrm{sp} 3)-\mathrm{C}(\mathrm{sp} 3)$ bond formation [1], as direct cyclopropanation of $\alpha$ cyano $\beta$-aryl alkanes by light-mediated single electron transfer between donor-acceptor pairs [2], in synthesis of cyanoacrylates by Knoevenagel condensation [3], in diastereoselective four-component synthesis of polysubstituted 2-piperidinones with three and four stereogenic centers [4], diastereoselective synthesis of spiro[2.3]hexanes from methylenecyclopropane and cyanoalkenes [5], in study of microporous polyurethane material for size selective heterogeneous catalysis of the Knoevenagel reaction [6], in the regio- and stereo-selective synthesis of spiro pyrrolidine and pyrrolizidine derivatives [7], in pyrrolizinone synthesis through functionalized C-alkylpyrroles [8], in synthesis of new pyridinecarbonitriles from fluoro arylpropenones [9], in chiral urea-catalyzed enantioselective epoxidation of $\alpha, \beta$-unsaturated esters [10], in green synthetic methodology of (E)-2-cyano-3-aryl selective Knoevenagel adducts under microwave irradiation [11], in syntheses of highly substituted cyclohexanes and cyclopentenes via phosphine-catalyzed chemo- and diastereoselective $[2+2+2]$ and $[3+2]$ annulations of $\gamma$-methyl allenoates with doubly activated olefins [12], in studies of carbonyl function switches from reacting to activating in aza-Wittig reaction with nitriles [13], in studies of compartmentalization of incompatible polymers within metal-organic frameworks towards homogenization of heterogeneous hybrid catalysts for tandem reactions [14], in Knoevenagel condensation catalyzed by novel Nmm-based ionic liquids in water [15], in 
tunable cinchona-based thioureas-catalysed asymmetric epoxidation to glycidic ester derivatives [16], in synthesis and biological evaluation of spiro[acenaphthylene-1,2'pyrrolidine] derivatives as potent anti-infective agents [17]. Iodo ring-substituted styrenic compound is reported in synthesis of aryl nitriles via aerobic oxidative cleavage of aryl $\mathrm{C}=\mathrm{C}$ Bonds with $\left(\mathrm{NH}_{4}\right)_{2} \mathrm{CO}_{3}$ as the nitrogen source [18], in a study of the effects of complex structure on aryl iodide oxidative addition at bipyridyl-ligated gold(I) centers [19], as well as synthesis and evaluation of a novel library of alternating amphipathic copolymers to solubilize and study membrane proteins [20].

We have reported synthesis and styrene copolymerization a number of fluoro and iodomethoxy ring-disubstituted PCAs, such esters as methyl [21], propyl [22, 23], isopropyl [24], butyl [25], isobutyl [26], 2-methoxyethyl [27], and octyl [28].

Thus, in continuation of our investigation of novel PCA compounds we have prepared ringdisubstituted isobutyl PCA, $\mathrm{RPhCH}=\mathrm{C}(\mathrm{CN}) \mathrm{CO}_{2} \mathrm{CH}_{2} \mathrm{CH}\left(\mathrm{CH}_{3}\right)_{2}$, where $\mathrm{R}$ is 2-fluoro-3methoxy, 2-fluoro-4-methoxy, 2-fluoro-5-methoxy, 2-fluoro-6-methoxy, 3-fluoro-4methoxy, 4-fluoro-3-methoxy, 5-fluoro-2-methoxy, 3-iodo-4-methoxy, 5-iodo-2-methoxy and explored feasibility of their copolymerization with styrene. To the best of our knowledge, there have been no reports on either synthesis of these compounds, nor their copolymerization with styrene [29].

\section{Experimental}

2-Fluoro-3-methoxy, 2-fluoro-4-methoxy, 2-fluoro-5-methoxy, 2-fluoro-6-methoxy, 3fluoro-4-methoxy, 4-fluoro-3-methoxy, 5-fluoro-2-methoxy, 3-iodo-4-methoxy, 
5-iodo-2-methoxy benzaldehydes, isobutyl cyanoacetate, piperidine, styrene, 1,1'-

azobis(cyclohexanecarbonitrile) (ABCN), and toluene supplied from Sigma-Aldrich Co., were used as received. Instrumentation is described in [30].

\section{Results and discussion}

\subsection{Synthesis and characterization of isobutyl phenylcyanoacrylates}

All isobutyl phenylcyanoacrylates (IPCA) compounds were synthesized by Knoevenagel condensation [31] of appropriate benzaldehydes with isobutyl cyanoacetate, catalyzed by base, piperidine (Scheme 1).

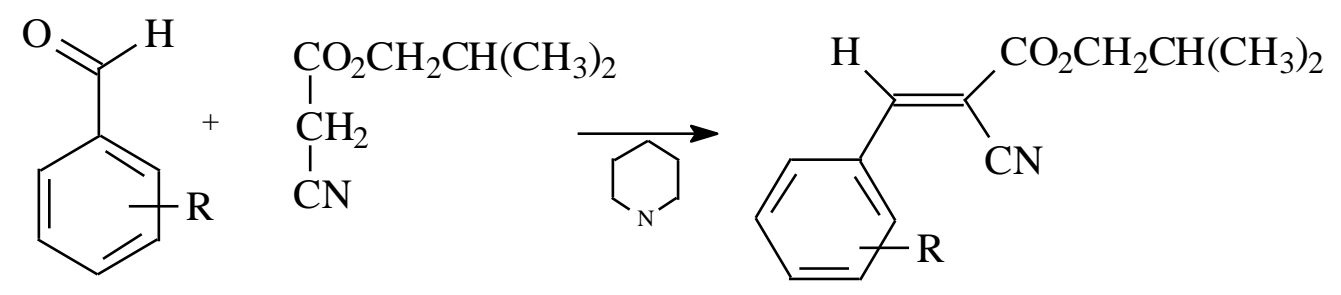

Scheme 1. Synthesis of isobutyl R-phenylcyanoacrylates, where R is 2-fluoro-3-methoxy, 2fluoro-4-methoxy, 2-fluoro-5-methoxy, 2-fluoro-6-methoxy, 3-fluoro-4-methoxy, 4-fluoro3-methoxy, 5-fluoro-2-methoxy, 3-iodo-4-methoxy, 5-iodo-2-methoxy.

The preparation procedure was essentially the same for all the monomers. In a typical synthesis, equimolar amounts of isobutyl cyanoacetate and an appropriate benzaldehyde were mixed in equimolar ratio in a $20 \mathrm{~mL}$ vial. A few drops of piperidine were added with stirring. The reactions was allowed to proceed $48 \mathrm{hrs}$ at r.t. The product of the reaction was isolated by filtration and purified by crystallization from 2-propanol. The condensation 
reaction proceeded smoothly, yielding products, which were purified by conventional techniques. Melting points of the compounds in crystalline state were measured by DSC.

The compounds were characterized by IR, ${ }^{1} \mathrm{H}$ and ${ }^{13} \mathrm{C}$ NMR spectroscopies. No stereochemical analysis of the novel ring-substituted IPCA was performed since no stereoisomers ( $E$ or/and $Z$ ) of known configuration were available.

\subsubsection{Isobutyl 2-fluoro-3-methoxyphenylcyanoacrylate}

Yield: $82.2 \%$; ${ }^{1} \mathrm{H}$ NMR: $\delta 8.6(\mathrm{~s}, 1 \mathrm{H}, \mathrm{CH}=), 8.0-7.0(\mathrm{~m}, 3 \mathrm{H}, \mathrm{Ph}), 4.1\left(\mathrm{~d}, 2 \mathrm{H}, \mathrm{CH}_{2}\right), 4.0(\mathrm{~s}$, $\left.3 \mathrm{H}, \mathrm{OCH}_{3}\right), 2.1(\mathrm{~m}, 1 \mathrm{H}, \mathrm{CH}), 0.9\left(\mathrm{~d}, 6 \mathrm{H}, \mathrm{CH}_{3}\right) ;{ }^{13} \mathrm{C} \mathrm{NMR}: \delta 162(\mathrm{C}=\mathrm{O}), 152(\mathrm{HC}=)$, 131, 110, $105(\mathrm{Ph}), 116(\mathrm{CN}), 103(\mathrm{C}=), 72\left(\mathrm{CH}_{2}\right), 54\left(\mathrm{OCH}_{3}\right), 27(\mathrm{CH}), 18\left(\mathrm{CH}_{3}\right)$; IR: $\left(\mathrm{cm}^{-1}\right) 2964(\mathrm{~m}, \mathrm{C}-\mathrm{H}), 2225(\mathrm{~m}, \mathrm{CN}), 1728$ (s, C=O), 1616 (s, C=C), $1277\left(\mathrm{~s}, \mathrm{C}-\mathrm{O}-\mathrm{CH}_{3}\right)$, 824, 719 (s, C-H out of plane). Anal. calcd. for $\mathrm{C}_{15} \mathrm{H}_{16} \mathrm{FNO}_{3}: \mathrm{C}, 64.97 ; \mathrm{H}, 5.82 ; \mathrm{N}, 5.05$; Found: C, 63.24; H, 5.85; N, 5.58.

\subsubsection{Isobutyl 2-fluoro-4-methoxyphenylcyanoacrylate}

Yield 78.6\%; mp 88.4 ${ }^{\circ} \mathrm{C} ;{ }^{1} \mathrm{H}$ NMR: $\delta 8.5$ (s, $\left.1 \mathrm{H}, \mathrm{CH}=\right), 7.3-6.6(\mathrm{~m}, 3 \mathrm{H}, \mathrm{Ph}), 4.1(\mathrm{~d}, 2 \mathrm{H}$, $\left.\mathrm{CH}_{2}\right), 3.9\left(\mathrm{~s}, 3 \mathrm{H}, \mathrm{OCH}_{3}\right), 2.1(\mathrm{~m}, 1 \mathrm{H}, \mathrm{CH}), 1.0\left(\mathrm{~d}, 6 \mathrm{H}, \mathrm{CH}_{3}\right) ;{ }^{13} \mathrm{C} \mathrm{NMR}: \delta 166(\mathrm{C}=\mathrm{O}), 152$ $(\mathrm{HC}=), 145,130,112,111(\mathrm{Ph}), 116(\mathrm{CN}), 102(\mathrm{C}=), 72\left(\mathrm{CH}_{2}\right), 56\left(\mathrm{OCH}_{3}\right), 28(\mathrm{CH}), 19$ $\left(\mathrm{CH}_{3}\right)_{2}$; IR: $\left(\mathrm{cm}^{-1}\right)$ 3002-2814 (m, C-H), 2218 (m, CN), 1717 (s, C=O), 1607 (s, C=C), 1257 (s, C-O-CH 3 ), 856, 752 (s, C-H out of plane). Anal. calcd. for $\mathrm{C}_{15} \mathrm{H}_{16} \mathrm{FNO}_{3}$ : C, 64.97; H, 5.82; N, 5.05; Found: C, 65.92; H, 5.92; N, 5.26.

\subsubsection{Isobutyl 2-fluoro-5-methoxyphenylcyanoacrylate}


Yield 91.3\%; ${ }^{1} \mathrm{H}$ NMR: $\delta 8.5$ (s, 1H, CH=), 8.0-7.3 (m, 3H, Ph), $4.2\left(\mathrm{~d}, 2 \mathrm{H}, \mathrm{CH}_{2}\right), 4.0$ (s, $\left.3 \mathrm{H}, \mathrm{OCH}_{3}\right), 2.1(\mathrm{~m}, 1 \mathrm{H}, \mathrm{CH}), 1.0\left(\mathrm{~d}, 6 \mathrm{H}, \mathrm{CH}_{3}\right) ;{ }^{13} \mathrm{C} \mathrm{NMR}: \delta 163(\mathrm{C}=\mathrm{O}), 151(\mathrm{HC}=)$, 132, 111, $107(\mathrm{Ph}), 115(\mathrm{CN}), 101(\mathrm{C}=), 71\left(\mathrm{CH}_{2}\right), 53\left(\mathrm{OCH}_{3}\right), 26(\mathrm{CH}), 18\left(\mathrm{CH}_{3}\right)$; IR: $\left(\mathrm{cm}^{-1}\right) 2982(\mathrm{~m}, \mathrm{C}-\mathrm{H}), 2224(\mathrm{~m}, \mathrm{CN}), 1730(\mathrm{~s}, \mathrm{C}=\mathrm{O}), 1610(\mathrm{~s}, \mathrm{C}=\mathrm{C}), 1271\left(\mathrm{~s}, \mathrm{C}-\mathrm{O}-\mathrm{CH}_{3}\right)$, 824, 802 (s, C-H out of plane). Anal. calcd. for $\mathrm{C}_{15} \mathrm{H}_{16} \mathrm{FNO}_{3}: \mathrm{C}, 64.97 ; \mathrm{H}, 5.82 ; \mathrm{N}, 5.05$; Found: C, 63.48; H, 5.88; N, 5.17.

\subsubsection{Isobutyl 2-fluoro-6-methoxyphenylcyanoacrylate}

Yield 86\%; ${ }^{1} \mathrm{H}$ NMR $\delta 8.3(\mathrm{~s}, 1 \mathrm{H}, \mathrm{CH}=), 7.4,6.7(\mathrm{~m}, 3 \mathrm{H}, \mathrm{Ph}), 3.9$ (d, 2H, $\left.\mathrm{CH}_{2}\right), 3.7$ (s, $\left.3 \mathrm{H}, \mathrm{OCH}_{3}\right), 2.0(\mathrm{~m}, 1 \mathrm{H}, \mathrm{CH}), 1.0\left(\mathrm{~d}, 6 \mathrm{H}, \mathrm{CH}_{3}\right) ;{ }^{13} \mathrm{C} \mathrm{NMR} \delta 162(\mathrm{C}=\mathrm{O}), 159(\mathrm{HC}=), 146$, 141, 134, 109, $108(\mathrm{Ph}), 114(\mathrm{CN}), 107(\mathrm{C}=), 73\left(\mathrm{CH}_{2}\right), 56\left(\mathrm{OCH}_{3}\right), 28(\mathrm{CH}), 19\left(\mathrm{CH}_{3}\right)$; IR (cm $\left.{ }^{-1}\right):$ 3050-2750 (m, C-H), 2233 (m, CN), 1728 (s, C=O), 1616 (s, C=C), 1261 (s, C-

O- $\mathrm{CH}_{3}$ ), 783, 764 (s, C-H out of plane). Anal. Calcd. for $\mathrm{C}_{15} \mathrm{H}_{16} \mathrm{FNO}_{3}$ : C, 64.97; H, 5.82;

N, 5.05; Found: C, 62.84; H, 5.84; N, 5.13.

\subsubsection{Isobutyl 3-fluoro-4-methoxyphenylcyanoacrylate}

Yield 66\%; mp 95.4 ${ }^{\circ} \mathrm{C} ;{ }^{1} \mathrm{H}$ NMR: $\delta 8.1$ (s, $\left.1 \mathrm{H}, \mathrm{CH}=\right), 7.9-6.9(\mathrm{~m}, 3 \mathrm{H}, \mathrm{Ph}), 4.1(\mathrm{~d}, 2 \mathrm{H}$, $\left.\mathrm{CH}_{2}\right), 4.0\left(\mathrm{~s}, 3 \mathrm{H}, \mathrm{OCH}_{3}\right), 2.1(\mathrm{~m}, 1 \mathrm{H}, \mathrm{CH}), 1.0\left(\mathrm{~d}, 6 \mathrm{H}, \mathrm{CH}_{3}\right) ;{ }^{13} \mathrm{C} \mathrm{NMR:} \delta 163(\mathrm{C}=\mathrm{O})$, $153(\mathrm{HC}=), 131,129,118,113(\mathrm{Ph}), 116(\mathrm{CN}), 101(\mathrm{C}=), 73\left(\mathrm{CH}_{2}\right), 56\left(\mathrm{OCH}_{3}\right), 28(\mathrm{CH})$, $19\left(\mathrm{CH}_{3}\right)$; IR: $\left(\mathrm{cm}^{-1}\right)$ 3005-2887 (m, C-H), 2226 (m, CN), 1717 (s, C=O), 1690 (s, C=C), 1304 (s, C-O-CH 3 ), 828, 752 (s, C-H out of plane). Anal. calcd. for $\mathrm{C}_{15} \mathrm{H}_{16} \mathrm{FNO}_{3}$ : C, 64.97; H, 5.82; N, 5.05; Found: C, 64.73; H, 5.83; N, 5.63.

\subsubsection{Isobutyl 4-fluoro-3-methoxyphenylcyanoacrylate}


Yield $82 \%$; mp $57.9^{\circ} \mathrm{C}$; ${ }^{1} \mathrm{H}$ NMR $\delta 8.2$ (s, $\left.1 \mathrm{H}, \mathrm{CH}=\right), 7.9,7.5,7.2(\mathrm{~m}, 3 \mathrm{H}, \mathrm{Ph}), 4.1$ (d, $\left.2 \mathrm{H}, \mathrm{CH}_{2}\right), 4.0\left(\mathrm{~s}, 3 \mathrm{H}, \mathrm{OCH}_{3}\right), 2.1(\mathrm{~m}, 1 \mathrm{H}, \mathrm{CH}), 1.0\left(\mathrm{~d}, 6 \mathrm{H}, \mathrm{CH}_{3}\right) ;{ }^{13} \mathrm{C} \mathrm{NMR} \delta 162(\mathrm{C}=\mathrm{O})$, $154(\mathrm{HC}=), 148,128,126,114(\mathrm{Ph}), 116(\mathrm{CN}), 102(\mathrm{C}=), 73\left(\mathrm{CH}_{2}\right), 57\left(\mathrm{OCH}_{3}\right), 28(\mathrm{CH})$, $19\left(\mathrm{CH}_{3}\right)_{2}$; IR (cm $\left.{ }^{-1}\right): 2963$ (m, C-H), 2220 (m, CN), 1724 (s, C=O), 1612 (s, C=C), 1250 (s, C-O-CH 3 ), 856, 814 (s, C-H out of plane). Anal. Calcd. for $\mathrm{C}_{15} \mathrm{H}_{16} \mathrm{FNO}_{3}: \mathrm{C}, 64.97 ; \mathrm{H}$, 5.82; N, 5.05; Found: C, 62.69; H, 5.99; N, 5.23.

\subsubsection{Isobutyl 5-fluoro-2-methoxyphenylcyanoacrylate}

Yield $89 \%$; mp $74.8^{\circ} \mathrm{C} ;{ }^{1} \mathrm{H}$ NMR $\delta 8.7$ (s, $\left.1 \mathrm{H}, \mathrm{CH}=\right), 8.1,7.3,7.0(\mathrm{~m}, 3 \mathrm{H}, \mathrm{Ph}), 4.1$ (d, $\left.2 \mathrm{H}, \mathrm{CH}_{2}\right), 3.9\left(\mathrm{~s}, 3 \mathrm{H}, \mathrm{CH}_{3} \mathrm{O}\right), 2.1(\mathrm{~m}, 1 \mathrm{H}, \mathrm{CH}), 1.0\left(\mathrm{~d}, 6 \mathrm{H}, \mathrm{CH}_{3}\right) ;{ }^{13} \mathrm{C} \mathrm{NMR} \delta 162$ $(\mathrm{C}=\mathrm{O}), 153(\mathrm{HC}=), 142$ 135, 132, 131, $129(\mathrm{Ph}), 115(\mathrm{CN}), 103(\mathrm{C}=), 72\left(\mathrm{CH}_{2}\right), 56$

$\left(\mathrm{CH}_{3} \mathrm{O}\right), 28(\mathrm{CH}), 19\left(\mathrm{CH}_{3}\right)_{2}$; IR $\left(\mathrm{cm}^{-1}\right): 2904$ (m, C-H), 2225 (m, CN), $1718(\mathrm{~s}, \mathrm{C}=\mathrm{O})$, 1607 (s, C=C), 1227 (s, C-O-CH 3 ), 818, 755 (s, C-H out of plane). Anal. Calcd. for $\mathrm{C}_{15} \mathrm{H}_{16} \mathrm{FNO}_{3}$ : C, 64.97; H, 5.82; N, 5.05; Found: C, 64.31; H, 5.79; N, 5.14.

\subsubsection{Isobutyl 3-iodo-4-methoxyphenylcyanoacrylate}

Yield 92\%; mp $127.2^{\circ} \mathrm{C} ;{ }^{1} \mathrm{H}$ NMR $\delta 8.3(\mathrm{~s}, 1 \mathrm{H}, \mathrm{CH}=), 8.1,8.0,6.9$ (s, 3H, Ph), $4.1(\mathrm{~s}$, $\left.2 \mathrm{H}, \mathrm{CH}_{2}\right), 4.0\left(\mathrm{~s}, 3 \mathrm{H}, \mathrm{OCH}_{3}\right), 2.0(\mathrm{~m}, 1 \mathrm{H}, \mathrm{CH}), 1.0\left(\mathrm{~d}, 6 \mathrm{H},\left(\mathrm{CH}_{3}\right)_{2} ;{ }^{13} \mathrm{C} \mathrm{NMR} \delta 163\right.$ $(\mathrm{C}=\mathrm{O}), 153(\mathrm{HC}=), 143,133,126,111(\mathrm{Ph}), 116(\mathrm{CN}), 101(\mathrm{C}=), 73\left(\mathrm{CH}_{2}\right), 57\left(\mathrm{CH}_{3} \mathrm{O}\right)$, $28(\mathrm{CH}), 19\left(\mathrm{CH}_{3}\right)_{2}$; IR $\left(\mathrm{cm}^{-1}\right): 2959(\mathrm{~m}, \mathrm{C}-\mathrm{H}), 2226(\mathrm{~m}, \mathrm{CN}), 1715$ (s, C=O), 1583 (s, $\mathrm{C}=\mathrm{C}), 1263\left(\mathrm{~s}, \mathrm{C}-\mathrm{O}-\mathrm{CH}_{3}\right), 842,755$ (s, C-H out of plane). Anal. Calcd. for $\mathrm{C}_{15} \mathrm{H}_{16} \mathrm{INO}_{3}$ : C, 46.77; H, 4.19; N, 3.64; Found: C, 45.62; H, 4.18; N, 3.82.

\subsubsection{Isobutyl 5-iodo-2-methoxyphenylcyanoacrylate}


Yield 71\%; mp $88.9^{\circ} \mathrm{C} ;{ }^{1} \mathrm{H}$ NMR $\delta 8.5$ (s, $\left.1 \mathrm{H}, \mathrm{CH}=\right), 8.4,8.1,6.7$ (s, 3H, Ph), 4.1 (s, 2H, $\left.\mathrm{CH}_{2}\right), 3.9\left(\mathrm{~s}, 3 \mathrm{H}, \mathrm{OCH}_{3}\right), 2.0(\mathrm{~m}, 1 \mathrm{H}, \mathrm{CH}), 1.0\left(\mathrm{~d}, 6 \mathrm{H},\left(\mathrm{CH}_{3}\right)_{2} ;{ }^{13} \mathrm{C} \mathrm{NMR} \delta 162(\mathrm{C}=\mathrm{O})\right.$, $159(\mathrm{HC}=), 148,144,143,138,137,126,123(\mathrm{Ph}), 115(\mathrm{CN}), 104(\mathrm{C}=), 73\left(\mathrm{CH}_{2}\right), 56$

$\left(\mathrm{CH}_{3} \mathrm{O}\right), 28(\mathrm{CH}), 19\left(\mathrm{CH}_{3}\right)_{2}$; IR $\left(\mathrm{cm}^{-1}\right): 2962(\mathrm{~m}, \mathrm{C}-\mathrm{H}), 2224(\mathrm{~m}, \mathrm{CN}), 1726(\mathrm{~s}, \mathrm{C}=\mathrm{O})$, 1585 (s, C=C), 1475 (s, C-O-CH3), 846 (s, C-H out of plane). Anal. Calcd. for $\mathrm{C}_{15} \mathrm{H}_{16} \mathrm{INO}_{3}$ : C, 46.77; H, 4.19; N, 3.64; Found: C, 45.87; H, 4.34; N, 3.76.

\subsection{Synthesis and characterization of styrene - IPCA copolymers}

Copolymers of the styrene (ST) and the IPCA compounds, P(ST-co-IPCA) were prepared in 25-mL glass screw cap vials at ST/IPCA $=3(\mathrm{~mol})$ the monomer feed using $0.12 \mathrm{~mol} / \mathrm{L}$ of $\mathrm{ABCN}$ at an overall monomer concentration $2.44 \mathrm{~mol} / \mathrm{L}$ in $10 \mathrm{~mL}$ of toluene. The copolymerization was conducted at $70^{\circ} \mathrm{C}$. After a predetermined time, the mixture was cooled to room temperature, and precipitated dropwise in methanol. The composition of the copolymers was determined based on the nitrogen content. The novel synthesized IPCA compounds copolymerized readily with ST under free-radical conditions (Scheme 2) forming white flaky precipitates when their solutions were poured into methanol. The conversion of the copolymers was kept between 10 and $20 \%$ to minimize compositional drift (Table 1). 
Table 1. Copolymerization of isobutyl phenylcyanoacrylates with styrene.

\begin{tabular}{|c|c|c|c|c|}
\hline $\mathrm{R}$ & $\begin{array}{l}\text { Yield }^{\mathrm{a}} \\
(\mathrm{wt} \%)\end{array}$ & $\underset{(\mathrm{wt} \%)}{\mathrm{N}}$ & $\begin{array}{c}\mathrm{m}_{1} \text { in } \\
\text { copol. } \\
\text { (mol\%) }\end{array}$ & $\begin{array}{c}\mathrm{m}_{2} \text { in } \\
\text { copol. } \\
\text { (mol\%) }\end{array}$ \\
\hline 2-F-3- $\mathrm{CH}_{3} \mathrm{O}$ & 10.2 & 2.73 & 69.4 & 30.6 \\
\hline $2-\mathrm{F}-4-\mathrm{CH}_{3} \mathrm{O}$ & 12.1 & 2.20 & 77.6 & 22.4 \\
\hline $2-\mathrm{F}-5-\mathrm{CH}_{3} \mathrm{O}$ & 15.6 & 2.48 & 73.4 & 26.6 \\
\hline 2-F-6- $\mathrm{CH}_{3} \mathrm{O}$ & 11.5 & 2.54 & 72.5 & 27.5 \\
\hline $3-\mathrm{F}-4-\mathrm{CH}_{3} \mathrm{O}$ & 11.3 & 2.52 & 72.8 & 27.2 \\
\hline $4-\mathrm{F}-3-\mathrm{CH}_{3} \mathrm{O}$ & 17.1 & 2.63 & 71.1 & 28.9 \\
\hline $5-\mathrm{F}-2-\mathrm{CH}_{3} \mathrm{O}$ & 13.2 & 3.16 & 58.9 & 41.1 \\
\hline 3-I-4- $\mathrm{CH}_{3} \mathrm{O}$ & 14.2 & 2.07 & 73.7 & 26.3 \\
\hline $5-\mathrm{I}-2-\mathrm{CH}_{3} \mathrm{O}$ & 14.7 & 1.93 & 76.6 & 23.4 \\
\hline
\end{tabular}

Nitrogen elemental analysis showed that between 22.4 and $41.1 \mathrm{~mol} \%$ of IPCA is present in the copolymers, which is indicative of relatively high reactivity of the IPCA monomers towards ST radical which is typical of oxy ring-substituted different esters PCA. Since IPCA monomers do not homopolymerize, the most likely structure of the copolymers would be isolated IPCA monomer $(y=1)$ units alternating with short ST sequences $(x>$ 1) (Scheme 2).

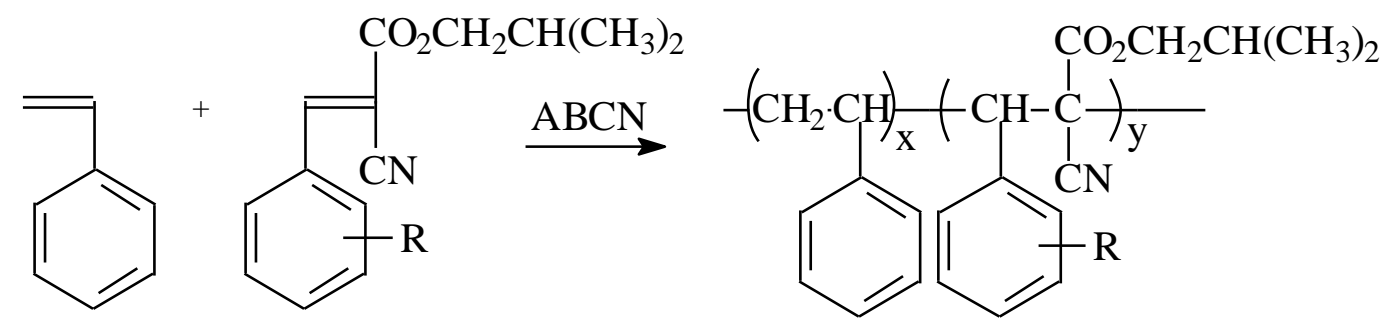

Scheme 2. Copolymerization of ST and the ring-substituted isobutyl phenylcyanoacrylates, $\mathrm{RPhCH}=\mathrm{C}(\mathrm{CN}) \mathrm{CO}_{2} \mathrm{CH}_{2} \mathrm{CH}\left(\mathrm{CH}_{3}\right)_{2}, \mathrm{R}=$ 2-fluoro-3-methoxy, 2fluoro-4-methoxy, 2-fluoro-5-methoxy, 2-fluoro-6-methoxy, 3-fluoro-4-methoxy, 4-fluoro3-methoxy, 5-fluoro-2-methoxy, 3-iodo-4-methoxy, 5-iodo-2-methoxy. 
The copolymers prepared in the present work are all soluble in ethyl acetate, THF, DMF and $\mathrm{CHCl}_{3}$ and insoluble in methanol, ethyl ether, and petroleum ether.

\section{Conclusions}

Novel fluoro and iodomethoxy ring-disubstituted isobutyl phenylcyanoacrylates were prepared and copolymerized with styrene. The compositions of the copolymers were calculated from nitrogen analysis.

\section{Acknowledgments}

The authors are grateful to acknowledge that the project was partly supported by Chicago Society of Coating Technology (CSCT).

\section{References}

1. Pentanidium-catalyzed direct assembly of vicinal all-carbon quaternary stereocenters through C(sp3)-C(sp3) bond formation. By Ban, Xu; Fan, Yifan; Kha, Tuan-Khoa; Lee, Richmond; Kee, Choon Wee; Jiang, Zhiyong; Tan, Choon-Hong. CCS Chemistry (2021), $3(10), 2192-2200$.

2. Direct Cyclopropanation of $\alpha$-Cyano $\beta$-Aryl Alkanes by Light-Mediated Single Electron Transfer Between Donor-Acceptor Pairs. Li, Jing; Lear, Martin J.; Hayashi, Yujiro. Chemistry - A European Journal (2021), 27(19), 5901-5905.

3. Facile Method for the Synthesis of Cyanoacrylates by Knoevenagel Condensation. Mabaso, Thabile; Shabalala, Nhlanhla Gracious; Kerru, Nagaraju; Jonnalagadda, Sreekantha B. Organic Preparations and Procedures International (2021), 53(1), 18-24. 
4. Highly diastereoselective four-component synthesis of polysubstituted 2-piperidinones with three and four stereogenic centers. Vereshchagin, Anatoly N.; Karpenko, Kirill A.; Elinson, Michail N.; Goloveshkin, Alexander S.; Dorofeeva, Evgeniya O.; Egorov, Mikhail P. Research on Chemical Intermediates (2020), 46(2), 1183-1199.

5. Diastereoselective Synthesis of Spiro[2.3]hexanes from Methylenecyclopropane and Cyanoalkenes Catalyzed by a Tin-Ate Complex. Suzuki, Itaru; Shimazu, Jun-ya; Tsunoi, Shinji; Shibata, Ikuya. European Journal of Organic Chemistry (2019), 2019(22), 36583661.

6. Microporous polyurethane material for size selective heterogeneous catalysis of the Knoevenagel reaction. Dey, Sandeep Kumar; de Sousa Amadeu, Nader; Janiak, Christoph. Chemical Communications (Cambridge, United Kingdom) (2016), 52(50), 7834-7837.

7. A highly efficient protocol for the regio- and stereo-selective synthesis of spiro pyrrolidine and pyrrolizidine derivatives by multicomponent reaction. Dandia, Anshu; Jain, Anuj K.; Laxkar, Ashok K.; Bhati, Dharmendra S. Tetrahedron Letters (2013), 54(24), 3180-3184.

8. An efficient synthetic route for pyrrolizinone synthesis through functionalized Calkylpyrroles. Unaleroglu, Canan; Tasgin, Dilek Isik; Aytac, Sertan; Temelli, Baris. Synthesis (2009), (19), 3243-3250.

9. New pyridinecarbonitriles from fluoro arylpropenones. Mishriky, N.; Asaad, F. M.;

Ibrahim, Y. A.; Girgis, A. S. Recueil des Travaux Chimiques des Pays-Bas (1994), 113(1), 35-9. 
10. Chiral urea-catalyzed enantioselective epoxidation of $\alpha, \beta$-unsaturated esters. Ji, Nan; Tian, Qinqin; Yang, Qingqing; Li, Minghua; He, Wei. Tetrahedron Letters (2021), 68, 152909.

11. Green Synthetic Methodology of (E)-2-cyano-3-aryl Selective Knoevenagel Adducts Under Microwave Irradiation. Quintero Jimenez, David Esteban; Zanin, Lucas Lima; Diniz, Luan Farinelli; Ellena, Javier; Meleiro Porto, Andre Luiz. Current Microwave Chemistry (2019), 6(1), 54-60.

12. Phosphine-Catalyzed Chemo- and Diastereoselective $[2+2+2]$ and $[3+2]$ Annulations of $\gamma$-Methyl Allenoates with Doubly Activated Olefins: Syntheses of Highly Substituted Cyclohexanes and Cyclopentenes.

Liu, Rongfang; Qin, Zifeng; Fan, Binbin; Li, Ruifeng; Zhou, Rong; He, Zhengjie. Journal of Organic Chemistry (2019), 84(19), 12490-12498.

13. aza-Wittig Reaction with Nitriles: How Carbonyl Function Switches from Reacting to Activating. Tukhtaev, Hamidulla B.; Ivanov, Konstantin L.; Bezzubov, Stanislav I.;

Cheshkov, Dmitry A.; Melnikov, Mikhail Ya.; Budynina, Ekaterina M. Organic Letters (2019), 21(4), 1087-1092.

14. Compartmentalization of incompatible polymers within metal-organic frameworks towards homogenization of heterogeneous hybrid catalysts for tandem reactions. Zhao, Jin-Hao; Yang, Yong; Che, Jin-Xin; Zuo, Jun; Li, Xiao-Hua; Hu, Yong-Zhou; Dong, Xiao-Wu; Gao, Liang; Liu, Xin-Yuan. Chemistry - A European Journal (2018), 24(39), 9903-9909. 
15. Knoevenagel condensation catalyzed by novel $\mathrm{Nmm}$-based ionic liquids in water. $\mathrm{Xu}$, Hao; Pan, Liyang; Fang, Xiaomin; Liu, Baoying; Zhang, Wenkai; Lu, Minghua; Xu, Yuanqing; Ding, Tao; Chang, Haibo. Tetrahedron Letters (2017), 58(24), 2360-2365.

16. Tunable Cinchona-Based Thioureas-Catalysed Asymmetric Epoxidation to Synthetically Important Glycidic Ester Derivatives. Meninno, Sara; Zullo, Luca;

Overgaard, Jacob; Lattanzi, Alessandra. Advanced Synthesis \& Catalysis (2017), 359(6), 913-918.

17. An efficient synthesis and biological evaluation of spiro[acenaphthylene-1,2'pyrrolidine] derivatives as potent anti-infective agents. Dandia, Anshu; Kumari, Sukhbeer; Soni, Pragya. European Chemical Bulletin (2013), 2(12), 1004-1008.

18. Synthesis of Aryl Nitriles via Aerobic Oxidative Cleavage of Aryl $\mathrm{C}=\mathrm{C}$ Bonds with (NH4)2CO3 as the Nitrogen Source. Zheng, Yi; Liu, Wenbo; Tian, Xinzhe; Ren, YunLai. Synlett (2022), 33(4), 376-380.

19. A Systematic Study of the Effects of Complex Structure on Aryl Iodide Oxidative Addition at Bipyridyl-Ligated Gold(I) Centers. Cadge, Jamie A.; Bower, John F.;

Russell, Christopher A. Angewandte Chemie, International Edition (2021), 60(47), 24976-24983.

20. Synthesis and evaluation of a novel library of alternating amphipathic copolymers to solubilize and study membrane proteins. Kopf, Adrian H.; Lijding, Odette; Elenbaas, Barend O. W.; Koorengevel, Martijn C.; van Walree, Cornelis A.; Killian, J. Antoinette. ChemRxiv (2021), 1-17. 
21. Novel Copolymers of Styrene. 11. Some Ring-Substituted Methyl 2-Cyano-3-phenyl2-propenates. G.B. Kharas, S.E. Chavez, A.N. Luna, E.E. Lusk, D.P. Mendez, D.S.

O'Rourke, C.S. Roat, S.G. Robinson, G.S. Stamelos, J.D. Schoenburg, D.H. Vinanzaca, S. Zermeno, M.P. O’Mara. J. Macromol. Sci. A51 (1), 1-5 (2014).

22. Novel Copolymers of Styrene. 7. Some Ring-Disubstituted Propyl 2-Cyano-3-Phenyl2-Propenoates. G.B. Kharas, V.A. Sloan-Lyon, M. Cieszynski, J.D.A. Manuel, S.L. Mei, J.B. Mubang Jr, P.E. Skorseth, Kaila E. Smith, N.M. Tassone, E. Tolberd, and D. Z.W. Yan. J. Macromol. Sci.A53(12) 729-733 (2016).

23. Novel Copolymers of Styrene. 8. Fluoro Ring-Disubstituted Propyl 2-Cyano-3Phenyl-2-Propenoates. G.B. Kharas, Y.L. Soto, E.L. Divino, M. Donovan, M.D. Dotson, S.A. Slazyk, D. Kishawi, J.H. Kittle, S. Logvinenko, B.M. McClarty, J.L. Pierce, and C.J. Smith. J. Macromol. Sci. A54(1) 1-5 (2017).

24. Synthesis and Styrene Copolymerization of Novel Fluoro and Oxy RingDisubstituted Isopropyl Phenylcyanoacrylates. A.R. Krause, H.E. Aynessazian, M.C. Gonzalez, A.M. Khan, L.R. Lieberman, M.H. McNicholas, N.M. Pelsi, Z.S. Pierard, S.M. Rocus, W.S. Schjerven, G.B. Kharas. (2019) ChemRxiv (20.11.2019). https://doi.org/10.26434/chemrxiv.10279751.v1

25. Novel Copolymers of Styrene. 9. Fluorine Ring-Disubstituted Butyl 2-Cyano-3Phenyl-2-Propenoates. G.B. Kharas, W.S. Schjerven, U.A. Baray, S. Chan, M.T. Cole, A.F. Haddad, J.A. Lucente, K.J. Patterson, A. Ralko, K.N. Reget, C.A. Shamblen, and E.M. Whitmore. J. Macromol. Sci. A53(4) 191-195 (2016). 
26. Synthesis and styrene copolymerization of novel fluoro and iodomethoxy ringdisubstituted isobutyl phenylcyanoacrylates. Umamah Alam, Magen A. Higgs, Kelly C. Ho, Kristin N. Huffman, Alexis L. Johnson, Paulina Kapica, Melanie A. Knight, Ashley Lehan, Christina Guyn, Briana N. Hryhorysak, Sara M. Rocus, William S. Schjerven and Gregory B. Kharas. ChemRxiv (17.09.2020) https://doi.org/10.26434/chemrxiv.11401347.v7

27. Synthesis and styrene copolymerization of novel trisubstituted ethylenes: 8 . Halogen ring-disubstituted 2-methoxyethyl phenylcyanoacrylates. Julianne Cappas, Emma Caraus, Sharad Chandra, Jacqueline A. Flores, Emily F. Jorgensen, Spozmai N. Khan, Emilia Kulka, Eiden Lami, Grace A. Mcgee, Gazmend Sadiku, Rami Sakka, Laith M. Shaban, Mariam Shaikh, Sara M. Rocus, William S. Schjerven, Gregory Kharas. ChemRxiv. Preprint. (01.02.2021). https://doi.org/10.26434/chemrxiv.13262660.v8

28. Synthesis and styrene copolymerization of novel trisubstituted ethylenes: 7. Fluoro, methyl and methoxy ring-disubstituted octyl phenylcyanoacrylates. Mahmoud D.

Awadallah, Izza A. Awan, Grace M. Carlino, Zachary K. Cherian, Sophia J. Dimonte, Madeline A. Purkey, Juvy L. Rabelas, Yash Y. Singapori, Bajeel Syeda, Victoria J.

Wisniewski, Sara M. Rocus, William S. Schjerven, and Gregory B. Kharas. ChemRxiv. Cambridge Open Engage Version 18 Jul 13, 2021. https://doi.org/10.33774/chemrxiv2021-k812v-v18

29. SciFinder, Structure Search, Feb 28, 2022.

30. Synthesis and styrene copolymerization of novel alkyl ring-substituted isobutyl 2cyano-3-phenyl-2-propenoates. Schjerven, William S.; Groy, Randi; Antonishina, 
Yekaterina S.; Black, Claire I.; Bolin, Madysen P.; Caniglia, John; Daroach, Deepak P.;

Farooqui, Farah T.; Feiz, Yalda; Garcia, Sam; Gasparini, Gabriella; Kharas, Gregory B. Abstracts of Papers, 259th ACS National Meeting \& Exposition, Philadelphia, PA, United States, March 22-26, (2020), POLY-0388.

31. Smith, M. B.; March, J. Addition to Carbon-Hetero Multiple Bonds, In March's Advanced Organic Chemistry, J. Wiley \& Sons: New York, Ch.16, 1225 (2001). 